\title{
Inhalt des 17. Bandes
}

\section{Heft 1}

Westwater, M. J., On the Renormalization of Feynman Integrals . . . . . . . . . 1

\section{Heft 2}

KUnszT, Z., and T. NAGY, Weak Decays of the Metastable Hadrons . . . . . . . . . 73

DkLOFF, A., Lambda-Nucleon Interaction . . . . . . . . . . . . . . . . . . . 129

\section{Heft 3}

Sebestyen, A., K. Szegö and K. Toth, An Introduction into the Higher Symmetries of

Regge-Poles . . . . . . . . . . . . . . . . . . 167

WIIHELMI, Z., Rotational and Quasi-rotational Spectra in Even-even Nuclei in the „New Region of Deformation" . . . . . . . . . . . . . . . . . . . . 189

BARTL, A., Die Summenregeln der Dispersionsmethode . . . . . . . . . . . . . . . 205

\section{Heft 4}

Nikrasson, G., Theory of First, Second and Zero Sound in One-Dimensional Crystals . 235

SkarkK, P., Beam Optics and Particle Transport . . . . . . . . . . . . 277

\section{Heft 5}

GERSCH, H.-U., Untersuchungen des Einfangmechanismus von Neutronen im Resonanzbereich 313

\section{Heft 6}

ScHNeIDER, H., Leptonische Zerfälle von $\Sigma$-Hyperonen und die Theorie von Cabibbo . . 393 Hegrrfeldt, G. C., and J. HenntG, Addendum . . . . . . . . . . . . . . . . . 463

\section{Heft 7}

Queen, N. M., Recent Applications of KN Forward Dispersion Relations and Sum Rules 467

KणCHOwICz, B., Neutrino and the Theory of Gravitation . . . . . . . . . 517

\section{Heft 8}

Malani, L., and G. Preparata, Algebra of Currents . . . . . . . . . . . . . . . . 537

\section{Heft 9}

KoHLER, R. H., Thermodynamics under Dynamic Forcing . . . . . . . . . . . . . 599

\section{Heft 10}

Barashenkov, V. S., K. K. Gudma and V. D. Toneev, Cross Sections for Fast Particles and Atomic Nuclei . . . . . . . . . . . . . . . . . . . . . . . 6883

\section{Heft 11}

Fonda, L., and G. C. GHIRARd, Lorentz Covariant Wave Functions and Equations for

Elementary Systems . . . . . . . . . . . . . . . . . . 727

\section{Heft 12}

Trum, U., Coherent Bremsstrahlung of Electrons in Crystals . . . . . . . . . 765 
Herausgeber: Prof. Dr. Frank Kaschluhn, Prof. Dr. Artur Losche, Prof. Dr. Rudolf Ritschl und Prof. Dr. Robert Rompe. Manuskripte sind zu richten an die Schriftleitung: Dr. Lutz Rothkirch, Sektion Physik der Humboldt-Universitat zu Berlin, 104 Berlin, Hessische Str. 2. Verlag: Akademie-Verlag GmbH, 108 Berlin, Leipziger Str. 3/4, Fernruf: 220441, Telex-Nr. 0112020, Postscheckkonto: Berlin 35021. Die Zeitschrift „Fortschritte der Physik" erscheint monatlich; Bezugspreis eines Heftes 10,- (Sonderpreis für die DDR 6,- M). Bestellnummer dieses Bandes: 1027/17. - Satz und Druck: VEB Druckhaus „Maxim Gorki“, 74 Altenburg, Bez. Leipzig, Carl-von-Ossletzky-Str. 30/31. - Veroffentlicht unter der Lizenznummer 1324 des Presseamtes beim Vorsitzenden des Ministerrates der Deutschen Demokratlschen Republik. 


\section{FORTSCHRITTE DER}

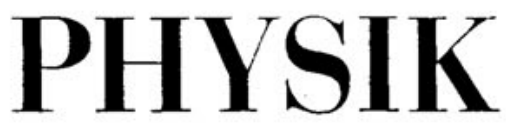

HERAUSGEGEBEN IM AUFTRAGE DER PHYSIKALISCHEN GESELLSCHAFT IN DER

DEUTSCHEN DEMOKRATISCHEN REPUBLIK

VON F. KASCHLUHN, A. LÖSCHE, R. RITSCHL UND R. ROMPE

BAND $17 \cdot$ HEFT $1 \cdot 1969$

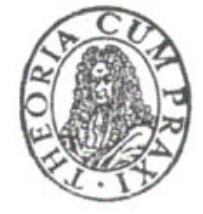

A K A D E M I E - V E R L A G • B E R L I N 


\section{Einführung in die Grundlagen der Quantentheorie}

Von Prof. Dr. E. FICK, Darmstadt

1968. 489 Seiten mit 95 Abbildungen

$16,5 \mathrm{~cm} \times 23,0 \mathrm{~cm} \cdot$ Kunstleder 57,- M (Sonderpreis für die DDR 48,- M)

In dem Hochschullehrbuch vermittelt der Verfasser eine den modernen Auffassungen und Erfordernissen entsprechende Darstellung der Quantentheorie.

Aus dem Inhalt: Die Vektoren des unitären Raumes · Lineare Operatoren · Der Produktraum . Geschichtliche Entwicklung und grundlegende Experimente zur Dualität Welle-Korpuskel Das Schrödingersche Materiefeld und seine Observablen · Stationäre Lösungen der Schrödinger gleichung · Die Dynamik des Schrödingerschen Materiefeldes · Hamiltonformalismus für das Materiefeld · Formulierung und Interpretierung der Quantentheorie · Spezielle Systeme

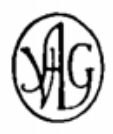

AKADEMISCHE VERLAGSGESELLSCHAFT GEEST \& PORTIG K.-G., LEIPZIG

\section{BEZUGSMOGLICHKEITEN}

Sämtliche Veröffentlichungen unseres Verlages sind durch jede Buchhandlung im In- und Ausland zu beziehen. Falls keine Bezugsmöglichkeit vorhanden ist, wende man sich

in der Deutschen Demokratischen Republik an den AKADEMIE-VERLAG, GmbH, DDR-108 Berlin, Leipziger Straße 3-4

in der Deutschen Bundesrepublik an KUNST UND WISSEN, Erich Bieber, 7 Stuttgart 1, Wilhelmstraße 4-6

in Osterreich an den GLOBUS-Buchvertrieb, Wien I, Salzgries 16

in Nord- und Südamerika an Gordon and Breach Science Publishers, Inc., 150 Fifth Avenue, New York, N. Y. 10011 U.S.A.

im sozialistischen Ausland an die Buchhandlungen für fremdsprachige Literatur bzw. den zuständigen Postzeitungsvertrieb

bei Wohnsitz im übrigen Ausland

an den Deutschen Buch-Export und -Import GmbH, DDR-701 Leipzig, Leninstraße 16. Auf Wunsch sendet der AKADEMIE-VERLAG Interessenten bei Bekanntgabe der Anschrift und Fachgebiete unverbindlich Informationen über lieferbare und kommende Veröffentlichungen und gibt auch Bezugsquellen im In- und Ausland bekannt. 